\title{
Synthetic Analogs of Curcumin Modulate the Function of Multidrug Resistance-Linked ATP-Binding Cassette Transporter ABCG2
}

\author{
Megumi Murakami, Shinobu Ohnuma, Michihiro Fukuda, Eduardo E. Chufan, Katsuyoshi Kudoh, \\ Keigo Kanehara, Norihiko Sugisawa, Masaharu Ishida, Takeshi Naitoh, Hiroyuki Shibata, \\ Yoshiharu Iwabuchi, Suresh V. Ambudkar, and Michiaki Unno
}

Department of Surgery, Graduate School of Medicine (M.M., S.O., K.Ku., K.Ka., N.S., M.I., T.N., M.U.), and Laboratory of Synthetic Chemistry, Department of Organic Chemistry, Graduate School of Pharmaceutical Sciences (M.F., Y.I.), Tohoku University, Sendai, Japan; Laboratory of Cell Biology, Center for Cancer Research, National Cancer Institute, National Institutes of Health, Bethesda, Maryland (E.E.C., S.V.A.); and Department of Clinical Oncology, Akita University Graduate School of Medicine, Akita, Japan (H.S.)

Received March 24, 2017; accepted September 5, 2017

\begin{abstract}
Multidrug resistance (MDR) caused by the overexpression of ATPbinding cassette (ABC) transporters in cancer cells is a major obstacle in cancer chemotherapy. Previous studies have shown that curcumin, a natural product and a dietary constituent of turmeric, inhibits the function of MDR-related ABC transporters, including $A B C B 1, A B C C 1$, and especially ABCG2. However, the limited bioavailability of curcumin prevents its use for modulation of the function of these transporters in the clinical setting. In this study, we investigated the effects of 24 synthetic curcumin analogs with increased bioavailability on the transport function of ABCG2. The screening of the 24 synthetic analogs by means of flow cytometry revealed that four of the curcumin analogs (GO-Y030, GO-Y078, GO-Y168, and GO-Y172) significantly inhibited the efflux of the $A B C G 2$ substrates, mitoxantrone and pheophorbide $A$, from
\end{abstract}

ABCG2-overexpressing K562/breast cancer resistance protein (BCRP) cells. Biochemical analyses showed that GO-Y030, GO-Y078, and GO-Y172 stimulated the ATPase activity of ABCG2 at nanomolar concentrations and inhibited the photolabeling of ABCG2 with iodoarylazidoprazosin, suggesting that these analogs interact with the substrate-binding sites of ABCG2. In addition, when used in cytotoxicity assays, GO-Y030 and GO-Y078 were found to improve the sensitivity of the anticancer drug, SN-38, in K562/BCRP cells. Taken together, these results suggest that nontoxic synthetic curcumin analogs with increased bioavailability, especially GO-Y030 and GO-Y078, inhibit the function of ABCG2 by directly interacting at the substrate-binding site. These synthetic curcumin analogs could therefore be developed as potent modulators to overcome ABCG2-mediated MDR in cancer cells.

\section{Introduction}

Chemotherapy is one of the effective treatments for cancer patients. However, patients often develop simultaneous resistance to many functionally and structurally unrelated anticancer drugs, a phenomenon known as multidrug resistance (MDR). Overexpression of ATP-binding cassette $(\mathrm{ABC})$ transporters in cancer cells is one of the leading causes of MDR (Gottesman et al., 2002). ABC transporters, such as ABCB1 (P-glycoprotein), ABCG2 [breast cancer resistance protein (BCRP)], and ABCC1 (MRP1), often overexpress in cell membranes of cancer cells and efflux the anticancer drugs from the intercellular to the extracellular (Ueda, 2011). Several compounds have been studied for their inhibitory effect on $\mathrm{ABC}$ transporters. For example, verapamil and cyclosporine $\mathrm{A}$ were effective for inhibiting the function of ABCB1 in vitro (Goldberg et al., 1988; Hamada and Tsuruo, 1988). However, because of the toxic

This work was supported by grant-in-aid for scientific research from the Japan Society for the Promotion of Science [Grant JP 25861149]. E.E.C. and S.V.A. were supported by the Intramural Research Program of the National Institutes of Health, National Cancer Institute, Center for Cancer Research.

https://doi.org/10.1124/dmd.117.076000. dose of these compounds required for inhibition of $\mathrm{ABCB} 1$, they led to severe side effects in vivo and made clinical application impossible. There have been no inhibitors for $\mathrm{ABC}$ transporters that are suitable for clinical application.

Recently, natural products, which are less toxic to animals, have been focused on the development of the inhibitor (Chanmahasathien et al., 2011; Pitchakarn et al., 2012). Curcumin is a natural product and a dietary constituent of turmeric (Ammon and Wahl, 1991). It is a well studied phytochemical that has the potential to suppress the growth of many cancer cell lines (Sa and Das, 2008; Lu et al., 2013). It has been shown that curcumin and its metabolites or constituents reverse the drug resistance in cells expressed by $\mathrm{ABCB} 1, \mathrm{ABCC} 1$, and especially ABCG2 (Anuchapreeda et al., 2002; Chearwae et al., 2004, 2006a,b). However, the characteristics of curcumin, which include hydrophobicity, low absorption, and rapid metabolism, caused limitations in clinical application (Sharma et al., 2004; Garcea et al., 2005; Goel et al., 2008).

Ohori et al. (2006) synthesized new curcumin analogs to increase the growth-suppressive ability of cancer cells while keeping their low toxicity. More than 2000 species from their synthetic organic compound library were screened to find the compound which could suppress the growth of colon cancer cell line DLD-1. Among them, GO-Y035, an

ABBREVIATIONS: ABC, ATP-binding cassette; BCRP, breast cancer resistance protein; BXP-21, BCRP-specific monoclonal antibody; FBS, fetal bovine serum; IAAP, iodoarylazidoprazosin; IMDM, Iscove's modified Dulbecco's medium; Ko143, tetracyclic analogue of fungal toxin fumitremorgin C (FTC); MDR, multidrug resistance; MTS, 3-(4,5-dimethylthiazol-2-yl)-5-(3-carboxymethoxyphenyl)-2-(4-sulfophenyl)-2H-tetrazolium; PBS, phosphate-buffered saline; SN-38, 7-ethyl-10-hydroxy-CPT. 
analog of curcumin, showed a stronger growth inhibition of DLD-1. Then, more than 50 curcumin analogs were synthesized by referring to the chemical structure of GO-Y035. Several derivatives showed a higher ability than curcumin to induce apoptosis in different cancer cells. These derivatives also decreased the expression of oncoproteins, such as $\beta$-catenin, Ki-ras, cyclin D1, and ErbB-2. An analog named GO-Y030 prevented adenoma formation in the familial adenomatous polyposis mouse model without any apparent toxicity (Shibata et al., 2009). Furthermore, GO-Y030 and another analog named GO-Y078, which has higher solubility than curcumin, upregulated apoptosis-related proteins such as TP-53 and DR5 (Kudo et al., 2011). Therefore, curcumin analogs have a more potent anticancer effect than curcumin. However, the role of curcumin analogs in the function of the MDR-related ABC transporter is largely unknown.

In this research, we studied the effect of curcumin analogs on ABCG2, because its transporter function was inhibited mostly by curcumin in a previous report (Limtrakul et al., 2007). We screened 24 curcumin analogs by transport assay and detected four curcumin analogs as potent inhibitors of $\mathrm{ABCG} 2$, then these four curcumin analogs were biochemically analyzed. These results suggested that nontoxic synthetic curcumin analogs might be useful to reverse ABCG2-mediated MDR.

\section{Materials and Methods}

Chemicals. RPMI 1640, Iscove's modified Dulbecco's medium (IMDM), fetal bovine serum (FBS), penicillin-streptomycin, curcumin, mitoxantrone, and pheophorbide A were purchased from Sigma-Aldrich (St. Louis, MO). Ko143 was purchased from Tocris Bioscience (Bristol, UK). SN-38 was purchased from Wako (Tokyo, Japan). Curcumin analogs (GO-Y030, GO-Y078, and other 22 analogs), synthesized as described previously (Ohori et al., 2006; Kohyama et al., 2015), were a generous gift from Dr. Shibata H. (Akita University, Akita, Japan) and Iwabuchi Y. (Tohoku University, Miyagi, Japan). Phosphate-buffered saline (PBS) and phycoerythrin-conjugated anti-human 5D3 antibody were procured by Thermo Fisher Science (Waltham, MA). BXP-21 monoclonal ABCG2 antibody was procured from Kamiya Biomedical (Seattle, WA). The MTS [3-(4,5-dimethylthiazol-2-yl)-5-(3-carboxymethoxyphenyl)-2-(4-sulfophenyl)$2 \mathrm{H}$-tetrazolium] viability kit was purchased from Promega (Madison, WI). $\left[{ }^{125} \mathrm{I}\right]$ iodoarylazidoprazosin (IAAP; $2200 \mathrm{Ci} / \mathrm{mmol}$ ) was obtained from PerkinElmer Life Science (Wellesley, MA).

Cell Lines. K562 human myelogenous leukemia cells and K562/BCRP, which overexpressed ABCG2 by transduction of ABCG2 into K562 cells with a retrovirus (Yanase et al., 2004), were cultured in RPMI 1640 supplemented with $10 \% \mathrm{FBS}$ and $5 \%$ penicillin-streptomycin at $37^{\circ} \mathrm{C}$ in $5 \% \mathrm{CO}_{2} . \mathrm{K} 562 / \mathrm{MDR}$, which overexpresses $\mathrm{ABCB} 1$, was used for comparison of ABCG2 expression. These cell lines were provided by Dr. Sugimoto Y. and Katayama K. (Keio University, Tokyo, Japan).

Reverse-Transcription Quantitative Polymerase Chain Reaction and Western Blotting. The expression of mRNA and protein of ABCB1 or ABCG2 on K562, K562/MDR, and K562/BCRP cell lines was determined by reversetranscription quantitative polymerase chain reaction and Western blotting. The isolation of RNA from K562 or K562/BCRP was performed using the RNeasy Mini Kit, purchased from Qiagen (Hilden, Germany). Reverse-transcription quantitative polymerase chain reaction and Western blotting were performed as described previously (Kobayashi et al., 2016). BXP-21 monoclonal antibody was used at 1:2000 and secondary anti-mouse antibody was used at 1:10,000 dilution for Western blotting.

Cell Surface Expression of ABCG2. The cell surface expression of ABCG2 on K562/BCRP cells was confirmed by flow cytometry. K562 and K562/BCRP cells were incubated with phycoerythrin-conjugated 5D3 antibody in IMDM for 45 minutes at $37^{\circ} \mathrm{C}$. The cells were washed with ice-cold IMDM with 5\% FBS and resuspended in PBS. The mean fluorescence intensity was calculated by BD FACS Verse and BD Suite software (Franklin Lakes, NJ).

Fluorescent Drug Accumulation Assay by Flow Cytometry. The effect of curcumin analogs on the cellular accumulation of mitoxantrone or pheophorbide A was determined by flow cytometry. K562 and K562/BCRP cells were incubated with mitoxantrone $(5 \mu \mathrm{M})$ for 45 minutes or pheophorbide $\mathrm{A}(5 \mu \mathrm{M})$

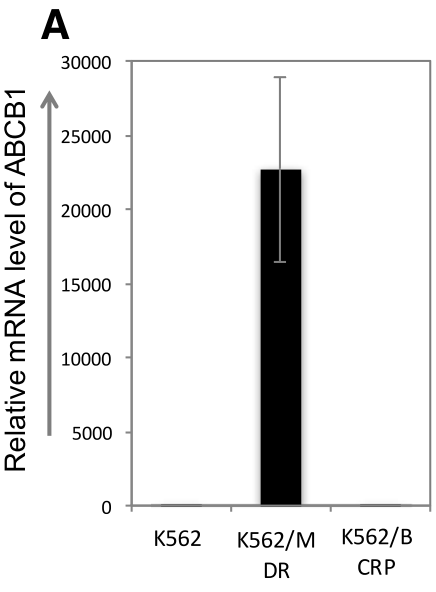

C

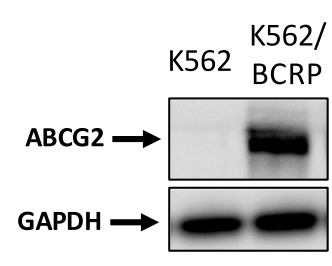

B

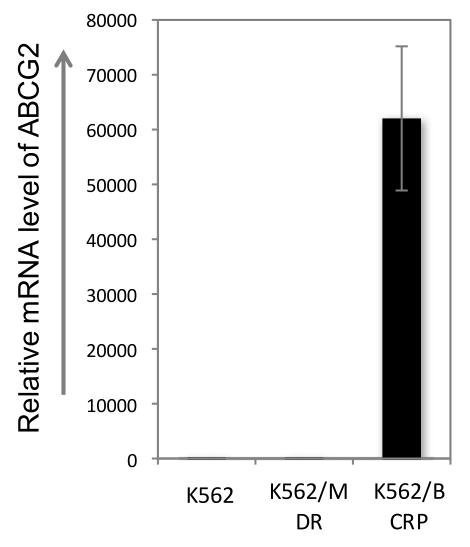

D

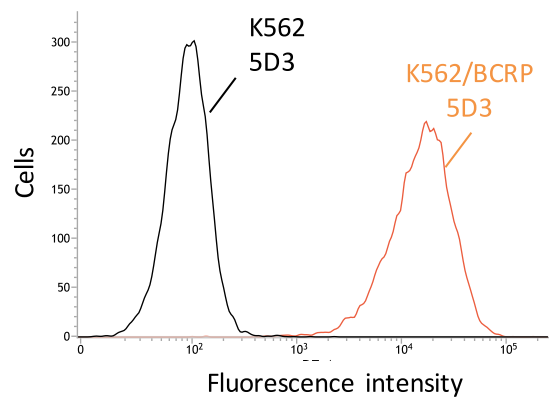

Fig. 1. ABCG2 expression of $\mathrm{K} 562$ and $\mathrm{K} 562 / \mathrm{BCRP}$ cell lines. (A and B) mRNA expression of ABCB1 (A) and ABCG2 (B) in K562, K562/MDR, and K562/BCRP cells by reverse-transcription quantitative polymerase chain reaction. Columns, mean $(n=3)$; bars, S.D. (C) Western blot analysis for ABCG2 using the anti-ABCG2 antibody BXP-21. (D) The cell surface expression of ABCG2 in K562/BCRP cells detected by monoclonal antibody 5D3. GAPDH, glyceraldehyde3-phosphate dehydrogenase. 
for 30 minutes at $37^{\circ} \mathrm{C}$ in IMDM with $5 \% \mathrm{FBS}$ in the presence or absence of curcumin analogs. Ko143 (10 $\mu \mathrm{M})$ was used as an inhibitor of ABCG2. The cells were washed with ice-cold PBS and resuspended in PBS. The samples were analyzed with mean fluorescence on BD FACS Verse or BD FACS Canto II with BD FACS Diva software (Franklin Rakes). The fluorescence of mitoxantrone or pheophorbide A was measured by a 700-nm band-pass filter. The $\mathrm{IC}_{50}$ values were calculated using GraphPad Prism 7 (GraphPad Software, La Jolla, CA).

ABCG2 Expression of K562/BCRP Cells Pretreated with Curcumin Analogs. The effect of curcumin analogs on ABCG2 protein expression in K562/BCRP was determined. K562/BCRP cells were cultured in $1 \mu \mathrm{M}$ curcumin

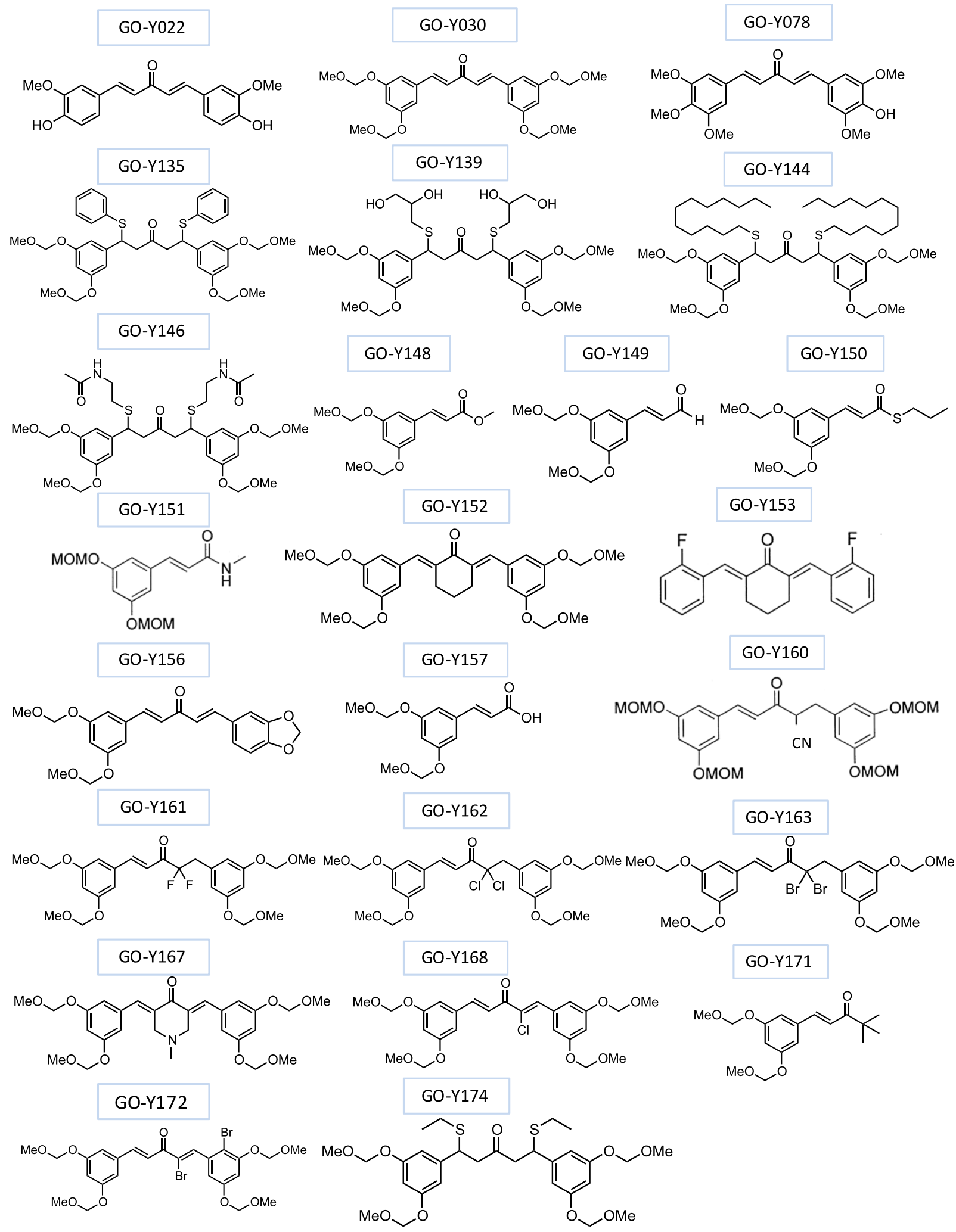

Fig. 2. The chemical structure of 24 curcumin analogs. 
A

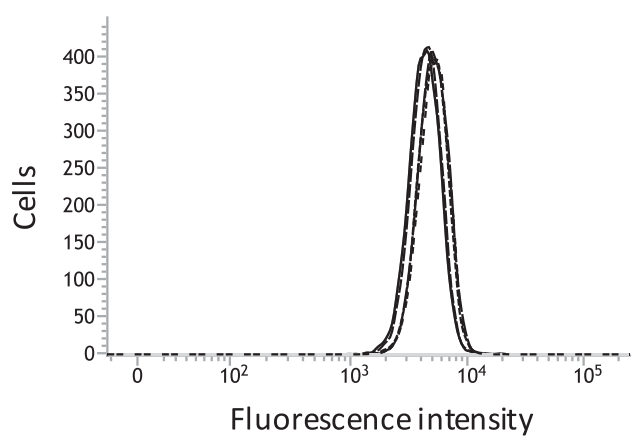

C

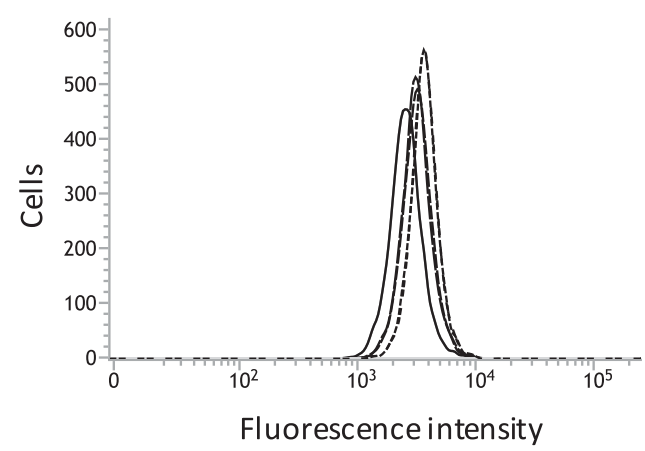

E

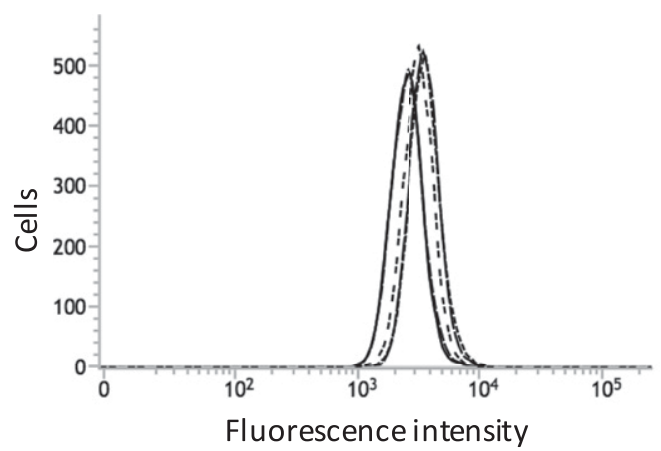

G

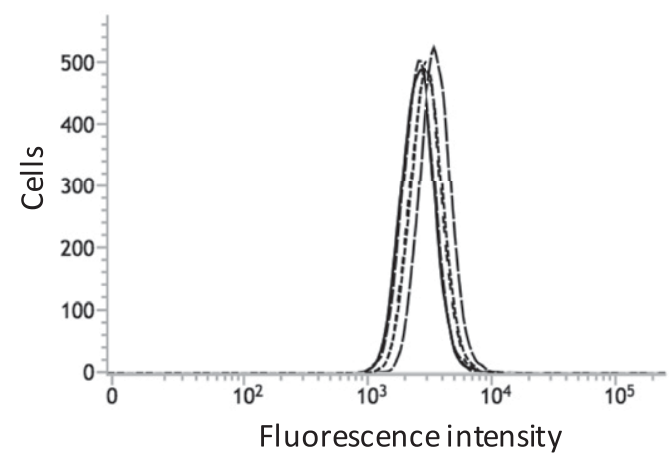

B

$20 \mu \mathrm{M}$

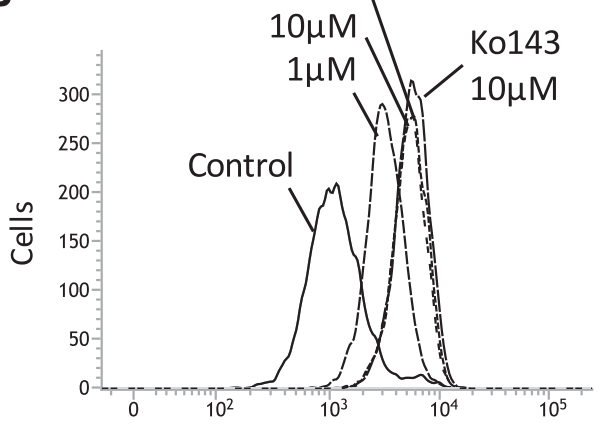

Fluorescence intensity

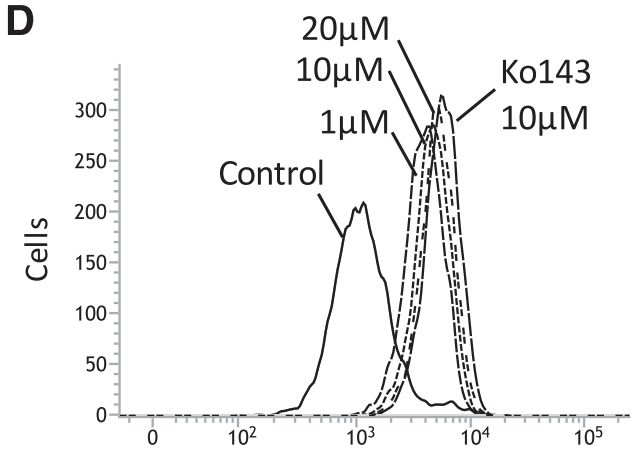

Fluorescence intensity

$\mathbf{F}$

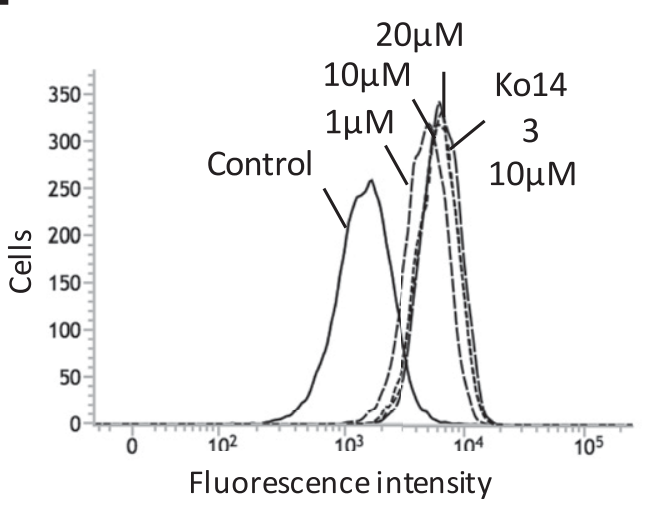

H

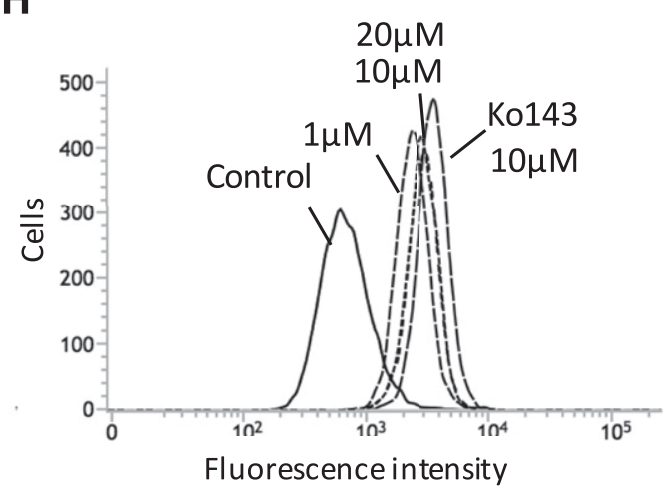

Fig. 3. Effect of curcumin analogs on mitoxantrone accumulation in K562 and K562/BCRP ABCG2-expressing cells. K562 cells (A, C, E, and G) and K562/BCRP cells $\left(\mathrm{B}, \mathrm{D}, \mathrm{F}\right.$, and $\mathrm{H}$ ) were incubated with $5 \mu \mathrm{M}$ mitoxantrone for 45 minutes at $37^{\circ} \mathrm{C}$ in the absence (control) or presence of various concentrations of curcumin analogs: GO-Y030 (A and B), GO-Y078 (C and D), GO-Y168 (E and F), and GO-Y172 (G and H). The cells were analyzed by flow cytometry. The histogram depicts fluorescence intensity ( $x$-axis) of control, 1,10 , and $20 \mu \mathrm{M}$ curcumin analogs. The result is from a representative experiment of three independent experiments. 
A

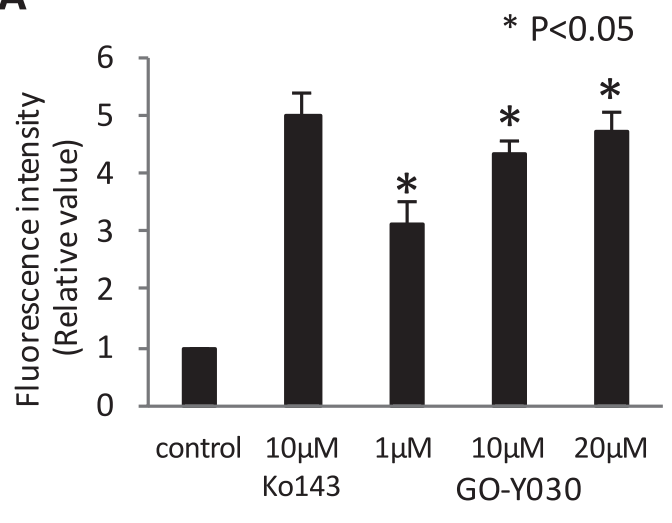

C

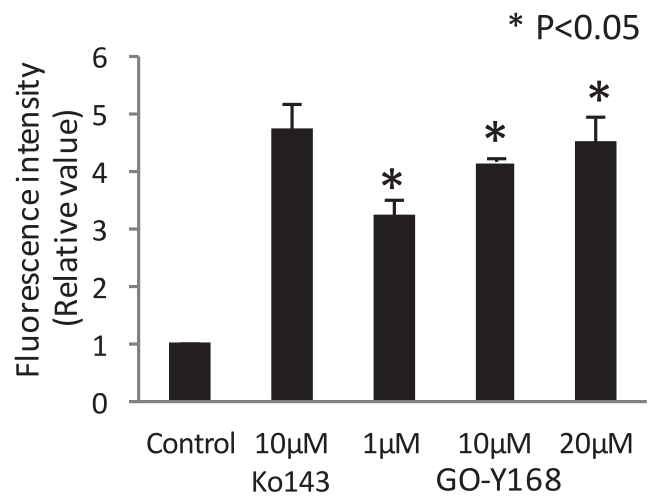

B

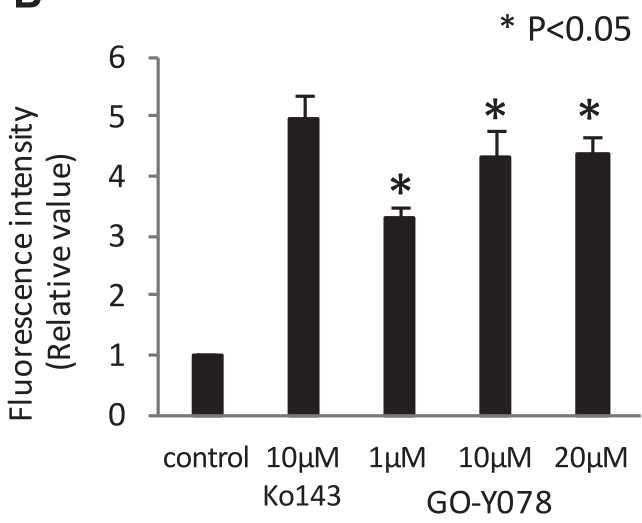

D

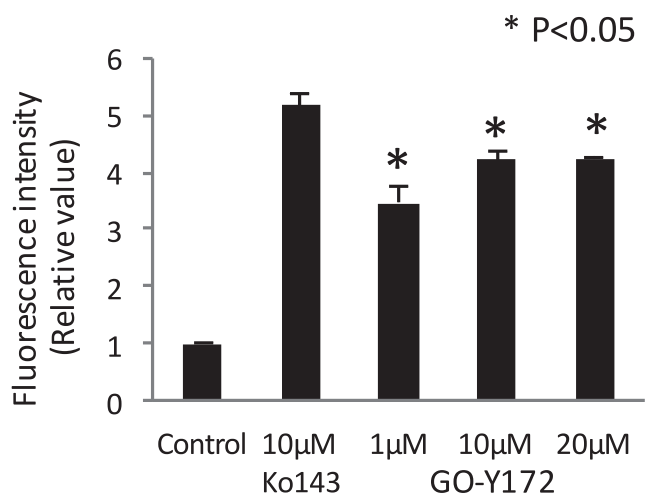

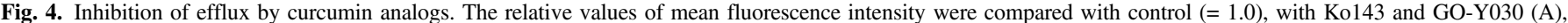
GO-Y078 (B), GO-Y168 (C), and GO-Y172 (D) in K562/BCRP cells. Columns, mean $(n=3)$; bars, S.D. $* P<0.05$ followed by Student's $t$ test.

or curcumin analogs for 3 days. The cells were washed with PBS, and the protein level of ABCG2 was determined by Western blotting as described earlier. The expression of ABCG2 was estimated by ImageQwant (Molecular Dynamics, Sunnyvale, CA).

ATPase Assay. The ABCG2-mediated ATP hydrolysis activity was determined by ATPase assay. The total membrane of ABCG2 expressing High Five insect cells, which were infected with recombinant baculovirus carrying the human ABCG2 cDNA, was used for ATPase assay as described previously (Shukla et al., 2007). ABCG2 expressing membrane protein $(6 \mu \mathrm{g} /$ tube) was incubated with varying concentrations of curcumin and curcumin analogs with or without sodium orthovanadate (Vi) in ATPase buffer. The reaction was started with $5 \mathrm{mM}$ ATP and incubated for 20 minutes at $37^{\circ} \mathrm{C}$. Sodium dodecyl sulfate solution $(5 \%, 0.1 \mathrm{ml} /$ tube $)$ was added to stop the reaction, and the amount of inorganic phosphate released was quantified by the colorimetric method as described previously (Ambudkar, 1998).

TABLE 1

Summary of inhibition of transport function of ABCG2 by curcumin and its analogs (percentage of control)

The inhibitory activity of transport function of ABCG2 was calculated by dividing the mean fluorescence intensity in the presence of $10 \mu \mathrm{M}$ curcumin or curcumin analogs by that in the presence of $10 \mu \mathrm{M}$ Kol43. The inhibition of transport function by $10 \mu \mathrm{M}$ Kol43 was considered as $100 \%$. The values are the mean \pm S.D. $(n=3)$ of three independent experiments.

\begin{tabular}{|c|c|c|c|c|c|}
\hline \multicolumn{2}{|c|}{ No Effect $(0 \%-25 \%)$} & \multicolumn{2}{|c|}{ Partial Inhibition (25\%-75\%) } & \multicolumn{2}{|c|}{ Significant Inhibition (75\%-100\%) } \\
\hline Compounds & $\%$ & Compounds & $\%$ & Compounds & $\%$ \\
\hline GO-Y139 & $1.6 \pm 2.6$ & GO-Y022 & 51.8 & GO-Y030 & $84.3 \pm 3.5$ \\
\hline GO-Y144 & $0.0 \pm 2.4$ & GO-Y135 & $26.6 \pm 2.7$ & GO-Y078 & $82.9 \pm 3.4$ \\
\hline GO-Y146 & $15.2 \pm 1.2$ & GO-Y150 & $28.2 \pm 6.0$ & GO-Y168 & $83.2 \pm 6.8$ \\
\hline GO-Y148 & 8.6 & GO-Y152 & $69.8 \pm 3.4$ & GO-Y172 & $77.4 \pm 1.2$ \\
\hline GO-Y149 & 9.2 & GO-Y153 & $30.5 \pm 9.9$ & Curcumin & $99.5 \pm 8.23$ \\
\hline GO-Y151 & 6.1 & GO-Y156 & $56.4 \pm 6.1$ & & \\
\hline GO-Y157 & 0.3 & GO-Y160 & $29.9 \pm 1.3$ & & \\
\hline \multirow[t]{5}{*}{ GO-Y171 } & $14.9 \pm 1.9$ & GO-Y161 & $44.4 \pm 3.2$ & & \\
\hline & & GO-Y162 & $51.5 \pm 9.0$ & & \\
\hline & & GO-Y163 & $58.7 \pm 2.2$ & & \\
\hline & & GO-Y167 & $52.0 \pm 15.9$ & & \\
\hline & & GO-Y174 & $37.1 \pm 2.2$ & & \\
\hline
\end{tabular}


TABLE 2

The $\mathrm{IC}_{50}$ values for inhibition of transport function of ABCG2 by curcumin, Ko143, GO-Y030, GO-Y078, GO-Y168, and GO-Y172

The values are the mean \pm S.D. of three independent experiments. The $I C_{50}$ values $(50 \%$ inhibitory concentration) were determined using concentrations of $0.05,0.1,0.25,0.5,1,5,10$ and $20 \mu \mathrm{M}$ of indicated compound, as described in Materials and Methods.

\begin{tabular}{lc}
\hline Compound & $\mathrm{IC}_{50}$ \\
\hline & $\mu M$ \\
Curcumin & $0.62 \pm 0.15$ \\
Ko143 & $0.19 \pm 0.11$ \\
GO-Y030 & $0.51 \pm 0.12$ \\
GO-Y078 & $0.31 \pm 0.09$ \\
GO-Y168 & $0.25 \pm 0.15$ \\
GO-Y172 & $0.37 \pm 0.17$ \\
\hline
\end{tabular}

Photolabeling of ABCG2 with $\left[{ }^{125}\right.$ I]Iodoarylazidoprazosin. The interaction of curcumin analogs with the substrate-binding site of ABCG2 was determined using the $\left[{ }^{125} \mathrm{I}\right] \mathrm{IAAP}$ photolabeling assay. Membranes of

A

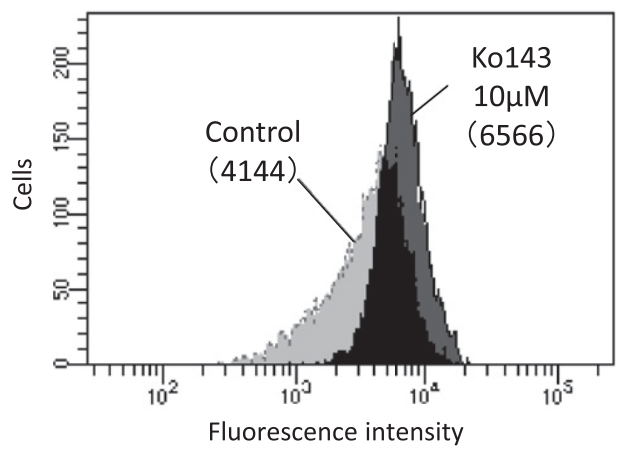

C

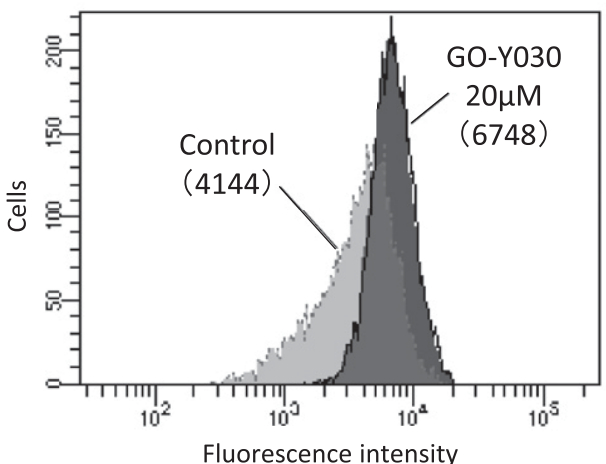

E

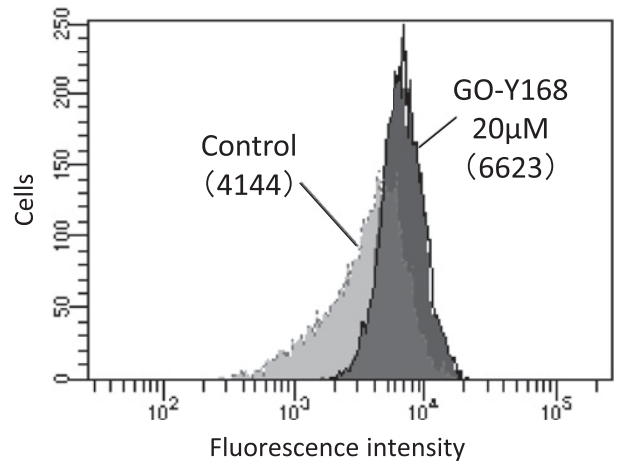

MCF7-FLV cells that overexpress ABCG2 were incubated with $10 \mu \mathrm{M}$ curcumin or curcumin analogs for 10 minutes at $37^{\circ} \mathrm{C}$. Then $3 \mathrm{nM}\left[{ }^{125} \mathrm{I}\right] \mathrm{IAAP}$ $(2200 \mathrm{Ci} / \mathrm{mmol})$ was added, and the mixture was irradiated with a $365 \mathrm{~nm}$ of UV light in ice-cold water for 10 minutes. An immunoprecipitation protocol was performed to ensure the reaction was specific for $\mathrm{ABCG} 2$ by adding BXP-21 antibody (Chearwae et al., 2006a). The samples were processed, and the photoaffinity labeling of $\left[{ }^{125}\right.$ I]IAAP with ABCG2 was estimated as described previously (Sauna et al., 2004).

Cytotoxicity Assay. The effect of curcumin analogs on anticancer drug cytotoxicity in K562 and K562/BCRP cells was determined by MTS assay. In brief, $5 \times 10^{5}$ cells were seeded into 96 wells and cultured overnight. Various concentrations of SN-38 either with or without $1 \mu \mathrm{M}$ curcumin analogs were added and incubated for 120 hours. After incubation, $20 \mu \mathrm{l}$ of MTS solution was added into each well and incubated for 4 hours. Absorbance was measured by using a Multiskan FC plate reader (Thermo Fisher Scientific) at $490 \mathrm{~nm}$. The percentage of cell survival was calculated as follows: (mean absorbance in test well - mean absorbance in blank well)/(mean absorbance in control well - mean absorbance in blank well) $\times 100$. The $\mathrm{IC}_{50}$ values were calculated by GraphPad Prism 7 .

B

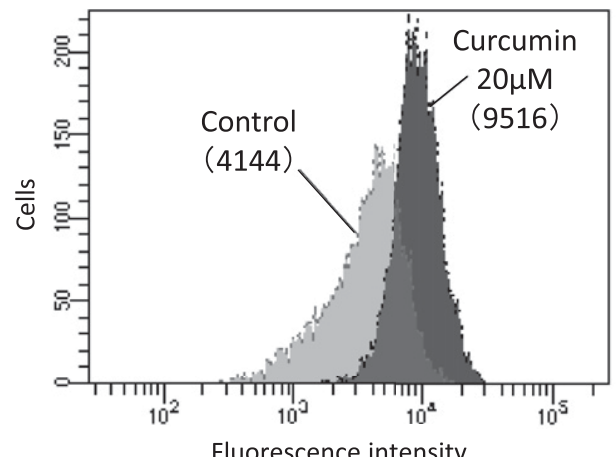

D

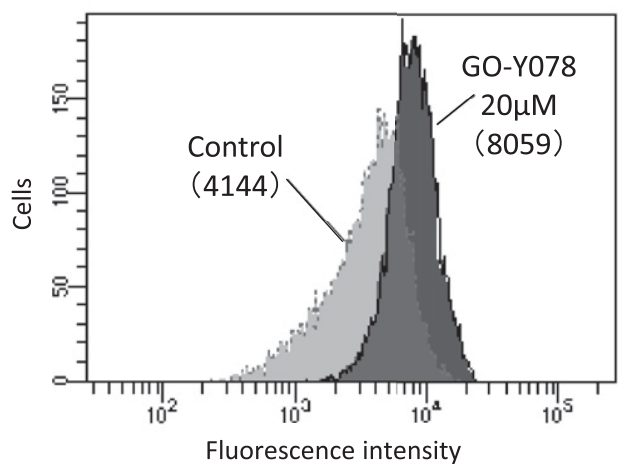

F

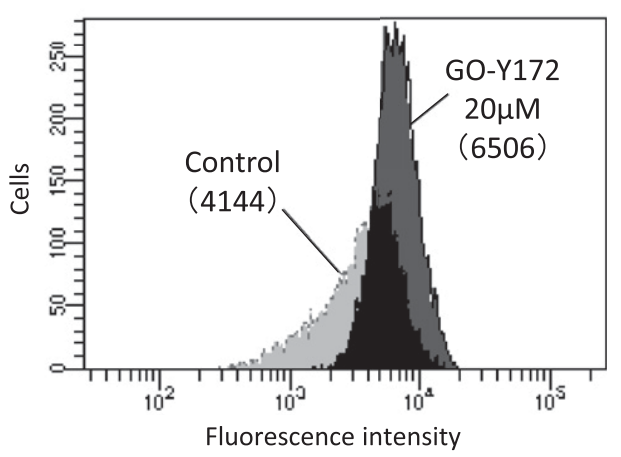

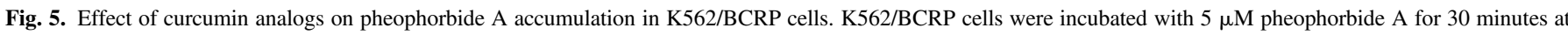

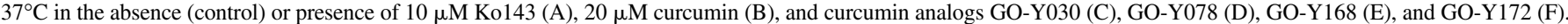
The cells were analyzed by flow cytometry. The values in parentheses on the histogram indicate mean fluorescence intensity. 
Statistical Analysis. Data are the mean \pm S.D. from at least three independent experiments. Differences between the means were analyzed by Student's $t$ test and Wilcoxon matched-pairs signed rank test. Results were considered to be statistically significant when $P<0.05$. Statistical analyses were performed using the JMP version 12.2 statistical software package (SAS International, Cary, NC) and GraphPad Prism 7.

\section{Results}

K562/BCRP Cells Overexpress Only ABCG2 Transporter. We confirmed ABCG2 expression in the K562/BCRP cell line. As shown in Fig. 1, A and B, ABCG2 mRNA was overexpressed in K562/BCRP cells; however, ABCB1 mRNA was not detected in K562/BCRP cells. Neither ABCB1 nor ABCG2 mRNA was detected in K562 cells (Fig. 1, $\mathrm{A}$ and $\mathrm{B})$. Consistent with the mRNA levels, the ABCG2 protein was also overexpressed in the K562/BCRP cell line (Fig. 1C). The expression of $\mathrm{ABCG} 2$ protein at the cell surface was determined by incubating with 5D3 anti-ABCG2 monoclonal antibody and detected by flow cytometry. K562/BCRP cells incubated with 5D3 ABCG2 antibody showed high fluorescence intensity compared with those with control parental K562 cells, suggesting that ABCG2 protein was overexpressed at the cell surface of K562/BCRP cells (Fig. 1D).

Screening for 24 Analogs of Curcumin on Transport Function of ABCG2. The screening for 24 synthetic analogs (Fig. 2) of curcumin by drug accumulation assay with flow cytometry was undertaken to determine their inhibitory effect on ABCG2-mediated function. Flow cytometry using fluorescent transport substrates of ABCG2 has been extensively used to measure transport function (Yanase et al., 2004). Cells overexpressing ABCG2 accumulate less fluorescent substrate, such as the typically used mitoxantrone, than parental cells not expressing ABCG2. Treatment of ABCG2-overexpressing cells with modulators that interact with $\mathrm{ABCG} 2$ decreases the efflux of the transport substrate and increases accumulation of the fluorescent substrates to levels seen in the parental cells. Figure 3B clearly shows that ABCG2-expressing K562/BCRP cells accumulate lower levels of mitoxantrone than the parental K562 cells shown in Fig. 3A. Treatment of the K562/BCRP cells with the ABCG2 modulator Ko143 increases mitoxantrone fluorescence to levels comparable to those observed in K562 cells (Figs. 3, A and B; Fig. 4A). When K562/BCRP cells were incubated with curcumin analog GO-Y030, the fluorescence intensity of mitoxantrone in K562/BCRP significantly increased in a dosedependent manner (Figs. 3B; Fig. 4A). The increase in the fluorescence signal with $20 \mu \mathrm{M}$ GO-Y030 was equivalent to that observed in the presence of $10 \mu \mathrm{M}$ Ko143. On the other hand, 1-20 $\mu \mathrm{M}$ GO-Y030 does not affect the fluorescence intensity of mitoxantrone in K562 (Fig. 3A). It is suggested that ABCG2-mediated efflux of mitoxantrone was blocked by GO-Y030. Furthermore, GO-Y078, GO-Y168, and GO-Y172 also showed an inhibitory effect on ABCG2-expressig K562/BCRP (Figs. 3, B, D, F, and H; Fig. 4, B-D).

We calculated the inhibitory activity by dividing the mean fluorescence intensity in the presence of $10 \mu \mathrm{M}$ curcumin analogs by that in the presence of $10 \mu \mathrm{M}$ Ko143, respectively, and summarized the data of inhibitory activity for 24 compounds in Table 1 . Out of 24 curcumin analogs, eight analogs (GO-Y139, GO-Y144, GO-Y146, GO-Y148, GO-Y149, GO-Y151, GO-Y157, and GO-Y171) showed lower inhibitory activity $(0 \%-15.2 \%)$. Twelve analogs (GO-Y022, GO-Y135, GO-Y150, GO-Y152, GO-Y153, GO-Y156, GO-Y160, GO-Y161, GO-Y162, GO-Y163, GO-Y167, and GO-Y174) showed partial inhibitory activity (26.6\%-73.8\%). Four curcumin analogs (GO-Y030, GO-Y078, GO-Y168, and GO-Y172) and curcumin showed significant inhibitory activity $(77.4 \%-99.5 \%)$ (Table 1$)$. The $50 \%$ inhibitory concentration $\left(\mathrm{IC}_{50}\right)$ values for the curcumin analogs that showed significant inhibitory activity was calculated. $\mathrm{IC}_{50}$ values for GO-Y030,
GO-Y078, GO-Y168, and GO-Y172 were $0.51 \pm 0.12,0.31 \pm 0.09$, $0.25 \pm 0.15$, and $0.37 \pm 0.17 \mu \mathrm{M}$, respectively (Table 2 ). These values were higher than that of Ko143 $(0.19 \pm 0.11 \mu \mathrm{M})$; however, they were lower than that for the curcumin $(0.62 \pm 0.15 \mu \mathrm{M})$ (Table 2). Furthermore, we checked the inhibitory effect of curcumin analogs with another ABCG2-specific fluorescent substrate, pheophorbide A, in an accumulation assay (Robey et al., 2004). GO-Y030, GO-Y078, GO-Y168, and GO-Y172 increased the fluorescence intensities of pheophorbide A 1.6-, 1.9-, 1.6-, and 1.6-fold, respectively, as compared with each control (Fig. 5, C-F). These values are almost comparable to that of Ko143 (1.6-fold stimulation) (Fig. 5A).

ABCG2 Expression in K562/BCRP Cells Is Not Affected by Exposure to Curcumin Analogs. To see the effect of curcumin analogs on ABCG2 protein expression in K562/BCRP, Western blotting was carried out. As shown in Fig. 6, 72-hour exposure of cells to $1 \mu \mathrm{M}$ curcumin, GO-Y030, GO-Y078, GO-Y168, and GO-Y172 did not change the expression of ABCG2 protein in K562/BCRP cells.

Because these four curcumin analogs were considered to be potent modulators of $\mathrm{ABCG} 2$ function, the following biochemical assays were carried out.

Modulation of ABCG2-Mediated ATP Hydrolysis by Curcumin Analogs. The substrate-stimulated ATP hydrolysis of ABCG2 by curcumin analogs was determined in the crude membranes of ABCG2-expressing High Five insect cells. As shown in Fig. 7A, curcumin stimulated ATPase activity of ABCG2; the concentration required for $50 \%$ stimulation $\left(\mathrm{EC}_{50}\right)$ was $14 \pm 0.002 \mathrm{nM}$ (Fig. 7A; Table 3). Three curcumin analogs, GO-Y030, GO-Y078, and GO-Y172, also stimulated ATP hydrolysis of ABCG2 in a concentration-dependent manner up to 2-fold of the basal activity at nanomolar concentrations;
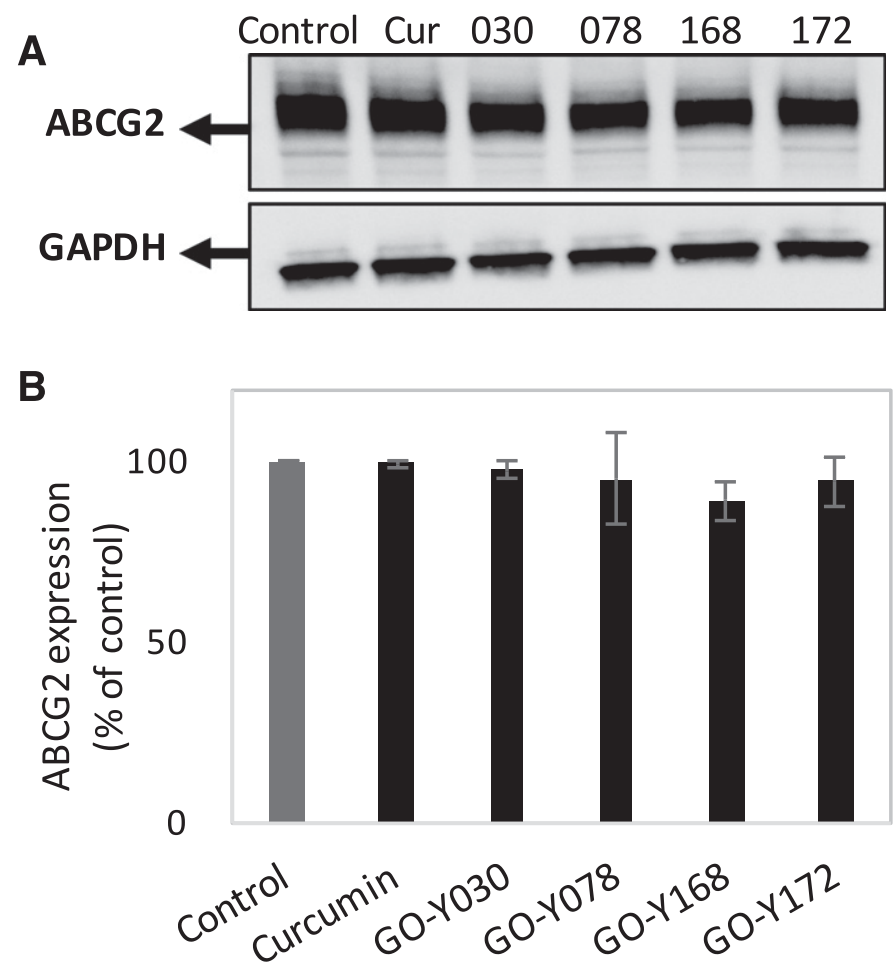

Fig. 6. Effect of curcumin and its analogs on ABCG2 protein expression of K562/BCRP cells. (A) The immunoblot shows the ABCG2 protein expression on K562/BCRP cells pretreated by incubating with $1 \mu \mathrm{M}$ curcumin or curcumin analogs for 72 hours. Arrow, position of the ABCG2 band; Cur, curcumin. (B) The ABCG2 protein expression in the absence (control, 100\%) or presence of curcumin and curcumin analogs. Columns, mean $(n=3)$; bars, S.D.; GAPDH, glyceraldehyde3-phosphate dehydrogenase. 
the $\mathrm{EC}_{50}$ values were $480 \pm 0.06 \mathrm{nM}$ for GO-Y030, $790 \pm 0.10 \mathrm{nM}$ for GO-Y078, and $930 \pm 0.12 \mathrm{nM}$ for GO-Y172 (Fig. 7, B-D; Table 3). Interestingly, GO-Y168 inhibited ATPase activity at lower concentrations $(0.25-0.5 \mu \mathrm{M})$ and stimulated at higher concentrations $(1-10 \mu \mathrm{M})$; the $\mathrm{EC}_{50}$ value was $3070 \pm 1.02 \mathrm{nM}$ (Fig. 7E; Table 3). These results suggest that GO-Y030, GO-Y078, GO-Y168, and GO-Y172 have high affinity with the substrate-binding site of ABCG2.

Effect of Curcumin Analogs on Photoaffinity Labeling of ABCG2 with [ ${ }^{\mathbf{1 2 5}}$ I]IAAP. To determine the interaction of curcumin analogs with the substrate-binding site of ABCG2, we monitored the effect of curcumin analogs on the photolabeling of ABCG2 with $\left[{ }^{125} \mathrm{I}\right]$ IAAP. The photoaffinity analog of prazosin, $\left[{ }^{125}\right.$ I]IAAP, is a transport substrate which binds to the substrate-binding site of ABCG2. It has been used extensively to study interactions at the substrate-binding sites.
Drug substrates of ABCG2 inhibit the photo-cross-linking of $\left[{ }^{125} \mathrm{I}\right]$ IAAP. Figure 8 clearly shows that each of the curcumin analogs, GO-Y030, GO-Y078, GO-Y168, and GO-Y172, at $10 \mu \mathrm{M}$ inhibited photolabeling of ABCG2 with $\left[{ }^{125}\right.$ I]IAAP similar to curcumin. These data demonstrate that these curcumin analogs interact directly with the substrate-binding site of ABCG2.

Effect of GO-Y030 and GO-Y078 on Cytotoxicity of SN-38 in K562/BCRP Cells. To confirm the effect of curcumin analogs on sensitivity of ABCG2-expressing cells to SN-38, which is an ABCG2 substrate and an active metabolite of irinotecan, cytotoxic assays were carried out. The $\mathrm{IC}_{50}$ for SN-38 in ABCG2-expresing K562/BCRP cells was 10-fold higher $\left(\mathrm{IC}_{50}=99.89 \pm 0.11 \mu \mathrm{M}\right)$ than that in $\mathrm{K} 562$ which has no ABCG2 $\left(\mathrm{IC}_{50}=9.92 \pm 0.18 \mu \mathrm{M}\right)($ Fig. 9; Table 4). However, $1 \mu \mathrm{M}$ of GO-Y030 increased cytotoxicity of SN-38 in K562/BCRP cells

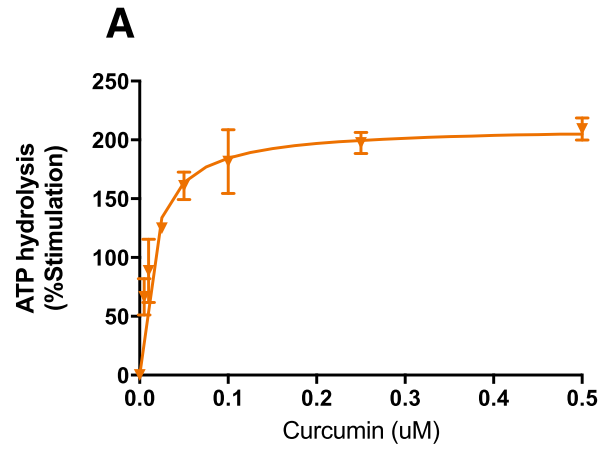

B

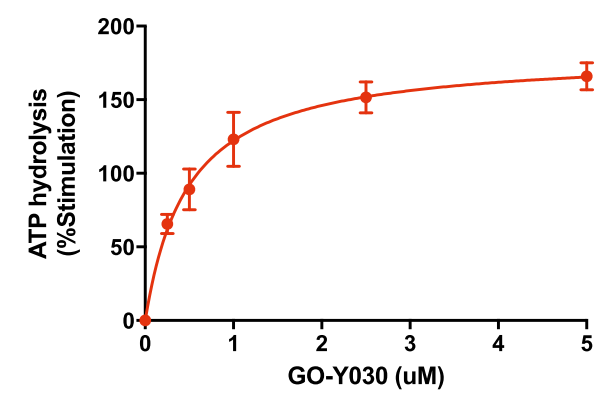

C

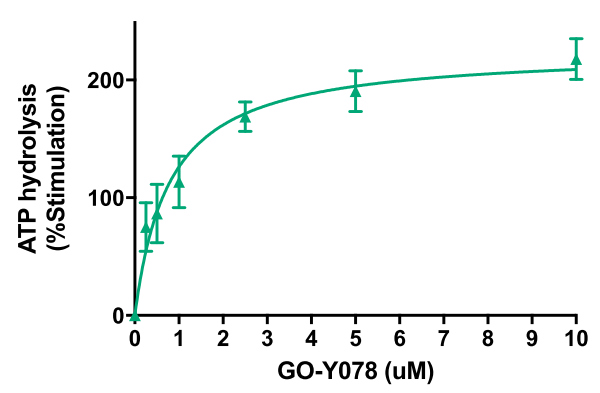

D
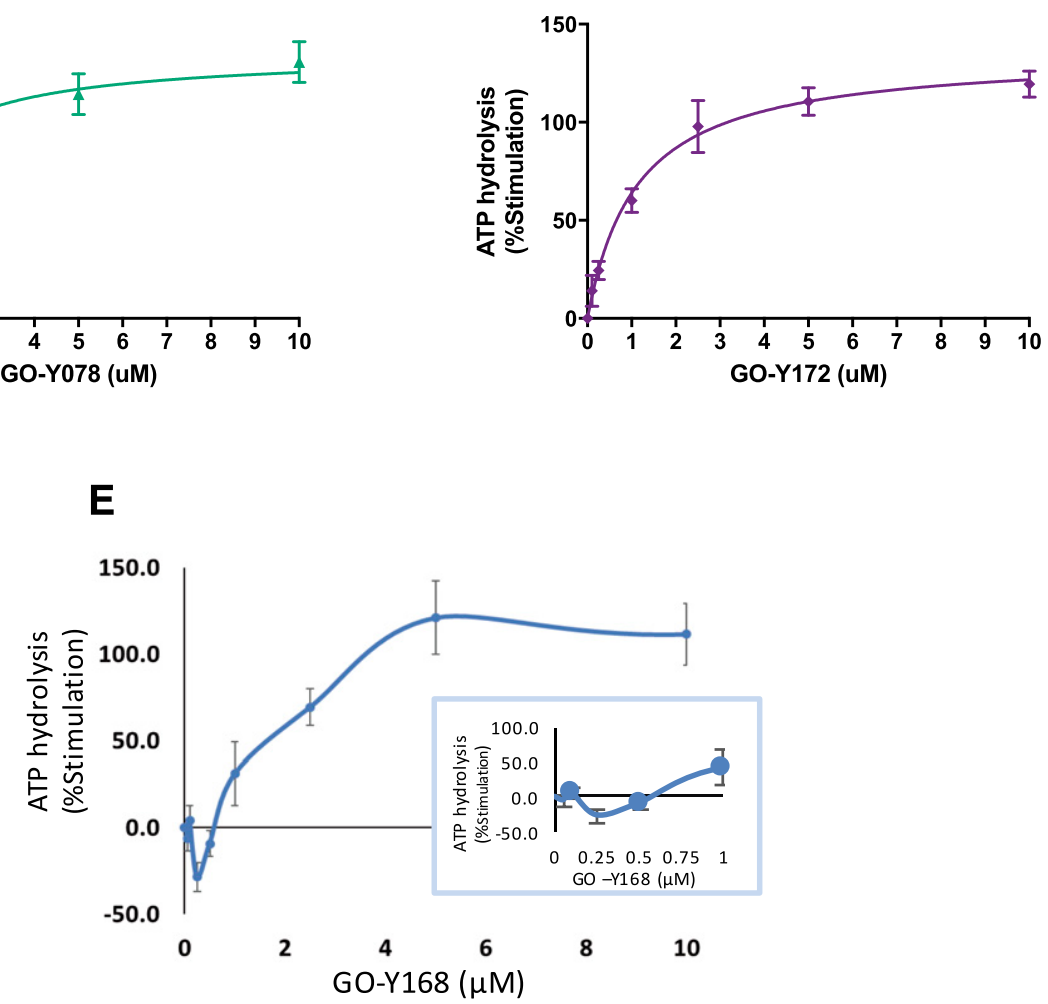

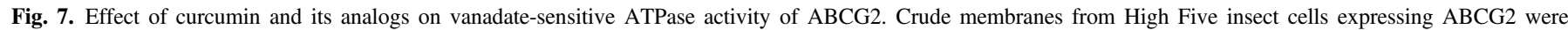

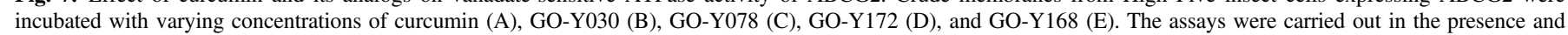

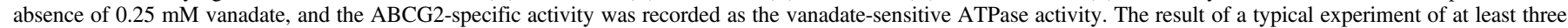
independent experiments is shown. 
TABLE 3

Stimulation of ATP hydrolysis of ABCG2 by curcumin and its analogs

The values are the mean \pm S.D. of three independent experiments. The ATPase assays were carried out as described in Materials and Methods, and EC 50 (concentration required for 50\% stimulation) values were determined.

\begin{tabular}{lc}
\hline Compounds & EC $_{50}$ \\
\hline & $n M$ \\
Curcumin & $14 \pm 0.002$ \\
GO-Y030 & $480 \pm 0.06$ \\
GO-Y078 & $790 \pm 0.10$ \\
GO-Y168 & $3070 \pm 1.02$ \\
GO-Y172 & $930 \pm 0.12$ \\
\hline
\end{tabular}

${ }^{a}$ Estimated value.

with a $\mathrm{IC}_{50}$ value $10.03 \pm 4.12 \mu \mathrm{M}$ (Fig. 9B; Table 4). Furthermore, $1 \mu \mathrm{M}$ GO-Y078 also increased cytotoxicity of SN-38 in K562/BCRP cells with an $\mathrm{IC}_{50}$ value of $11.71 \pm 4.14 \mu \mathrm{M}$ (Fig. 9D; Table 4). These $\mathrm{IC}_{50}$ values were approximately equal to those potentiated by $10 \mu \mathrm{M}$ Ko143 (Fig. 9, B and D; Table 4). However, the enhancement of cytotoxicity of SN-38 by GO-Y030 and GO-Y078 was not observed in K562 cells without expression of ABCG2 (Fig. 9, A and C). There were no effects of GO-Y168 and GO-Y172 on cytotoxicity of SN-38 in K562/BCRP cells (data not shown).

\section{Discussion}

Previous studies have indicated that curcumin, a natural product and a dietary constituent of turmeric, inhibits the function of MDR-linked $\mathrm{ABC}$ drug transporters, such as ABCB1, ABCC1, and ABCG2 (Anand et al., 2007; Limtrakul et al., 2007). However, the limited bioavailability of curcumin prevents its use as a modulator of the function of these transporters in a clinical setting (Sharma et al., 2004; Garcea et al., 2005). Numerous approaches have been developed to address the low bioavailability of curcumin, such as the use of adjuvants like piperine (Shoba et al., 1998), and the synthesis of liposomal curcumin (Li et al., 2005), curcumin nanoparticles (Bisht et al., 2007), and curcumin phospholipid complex (Marczylo et al., 2007). Furthermore, the development of structural analogs of curcumin is considered to be one of the most effective approaches. These synthetic curcumin analogs were developed in our institute and have been shown to exhibit greater bioavailability and anticancer effects than natural curcumin (Ohori et al., 2006). However, it remains unknown whether these synthetic analogs modulate the function of MDR-related $\mathrm{ABC}$ transporters.

The screening of 24 curcumin analogs on ABCG2-expressing K562/BCRP cells using flow cytometry revealed that four of the analogs (GO-Y030, GO-Y078, GO-Y168, and GO-Y172) were able to block the efflux of the ABCG2 substrate mitoxantrone. As shown in Fig. 3, GO-Y030, GO-Y078, GO-Y168, and GO-Y172 significantly inhibited the efflux of mitoxantrone in K562/BCRP cells, which have higher expression of $\mathrm{ABCG} 2$ and no expression of $\mathrm{ABCB} 1$ (Fig. 1, A and $\mathrm{B}$; Fig. 3). The $\mathrm{IC}_{50}$ values of these four analogs were almost equivalent to that of Ko143, which is a well known inhibitor of ABCG2 (Table 2). Furthermore, four curcumin analogs inhibited the efflux of pheophorbide A, which is specifically transported by ABCG2 (Fig. 5). In addition, Fig. 6 shows that four curcumin analogs had no effect on the expression of ABCG2 protein in K562/BCRP cells. Thus, these results suggest that analogs GO-Y030, GO-Y078, GO-Y168, and GO-Y172 can specifically inhibit ABCG2-mediated transport function without any alteration of expression of this transporter.

Subsequently, we carried out a biochemical investigation of how these analogs were able to modulate the ABCG2 function. The photolabeling of ABCG2 with [ ${ }^{125}$ I]IAAP was undertaken to determine
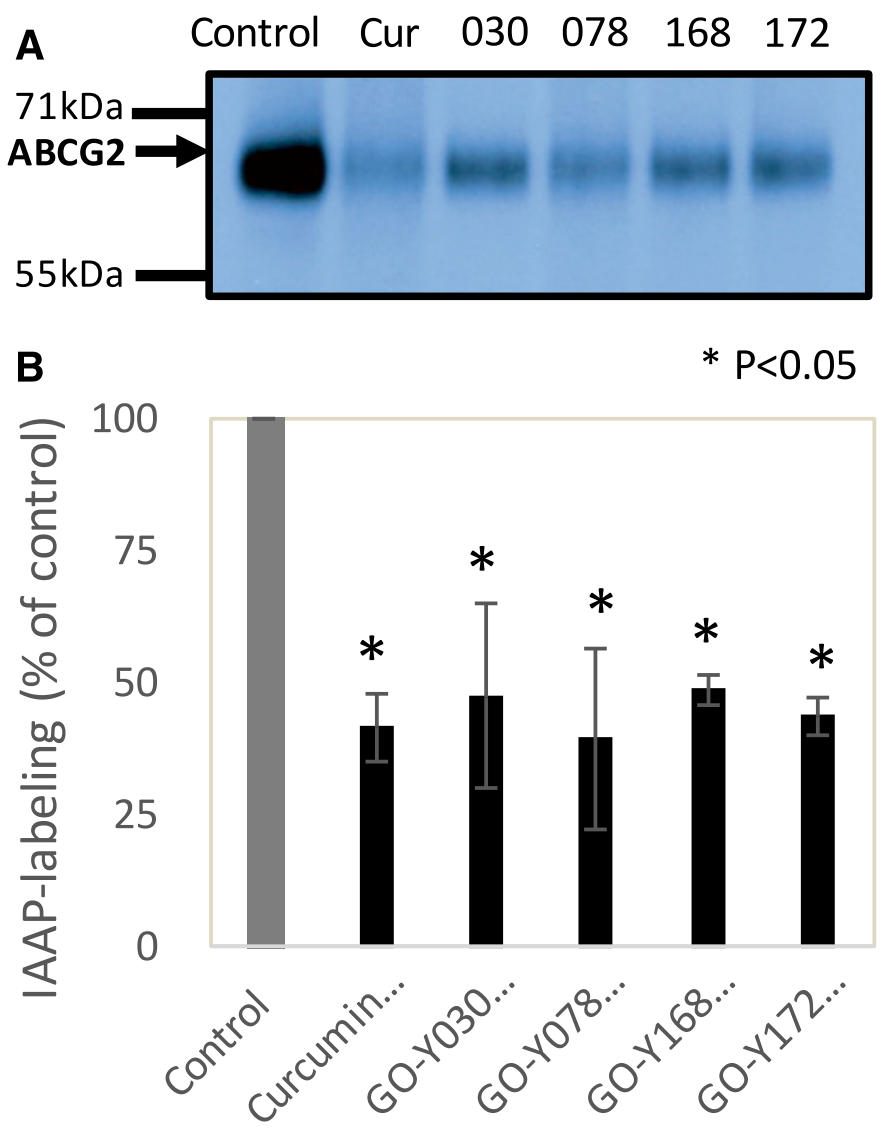

Fig. 8. Inhibition of $\left[{ }^{125}\right.$ I]IAAP labeling of human ABCG2 by curcumin and its analogs. Crude membranes from MCF7/FLV cells were incubated with $10 \mu \mathrm{M}$ curcumin and curcumin analogs at $37^{\circ} \mathrm{C}$ in $50 \mathrm{mM}$ Tris- $\mathrm{HCl}, \mathrm{pH} 7.5$, for 10 minutes IAAP (3 nM) was added and illuminated with a UV lamp $(365 \mathrm{~nm})$ for 10 minutes on ice-cold water. An immunoprecipitation protocol was performed to ensure the reaction was specific for ABCG2 by adding BXP-21 antibody. Following SDS-PAGE on a $7 \%$ Tris-acetate gel, gels were dried and exposed on X-ray at $-80^{\circ} \mathrm{C}$ for $8-15$ hours. The radioactivity incorporated into the ABCG2 band was quantified using the STORM 860 phosphorimager system (Molecular Dynamics, Sunnyvale, CA). (A) The autoradiogram showed incorporation of IAAP into the ABCG2 band in the presence $10 \mu \mathrm{M}$ curcumin and curcumin analogs. Arrow, position of the ABCG2 band; Cur, curcumin. (B) IAAP-labeling of ABCG2 with the absence (control, $100 \%$ ) or presence curcumin and curcumin analogs. Columns, mean $(n=3)$; bars, S.D.

the interaction of the curcumin analogs with the substrate-binding site. IAAP is a substrate of ABCG2, and it has been used extensively to study interactions at the substrate-binding sites of ABCG2. As shown in Fig. 8, GO-Y030, GO-Y078, GO-Y168, and GO-Y172 significantly inhibited the photolabeling of ABCG2 with $\left[{ }^{125}\right.$ I]IAAP, indicating that these curcumin analogs interact directly with the substrate-binding site of ABCG2. To verify further the interaction of the analogs with ABCG2, an ATPase assay was used. This is another useful assay for studying the interaction of transport substrates with ABCG2, since ATP hydrolysis is coupled with the transport function of this transporter (Glavinas et al., 2007; Gallus et al., 2014). The analogs were found to stimulate ATP hydrolysis in a dose-dependent manner. The previous finding also implies that these analogs interact with the substrate-binding site of ABCG2. Interestingly, GO-Y168 inhibited ATP hydrolysis at a lower concentration $(0.25-0.5 \mu \mathrm{M})$ and stimulated it a higher concentration $(1-10 \mu \mathrm{M})$ (Fig. 7E). To find out the role of the halogen atom of GO-Y168, ATPase assays were carried out using other curcumin analogs with a similar structure to GO-Y168. In these assays, the halogen atom chlorine $(\mathrm{Cl})$ was replaced with $\mathrm{CN}$ (GO-Y160), $\mathrm{F}_{2}$ (GO-Y161), $\mathrm{Cl}_{2}$ (GO-Y162), and $\mathrm{Br}_{2}$ (GO-Y163), respectively. However, 
A

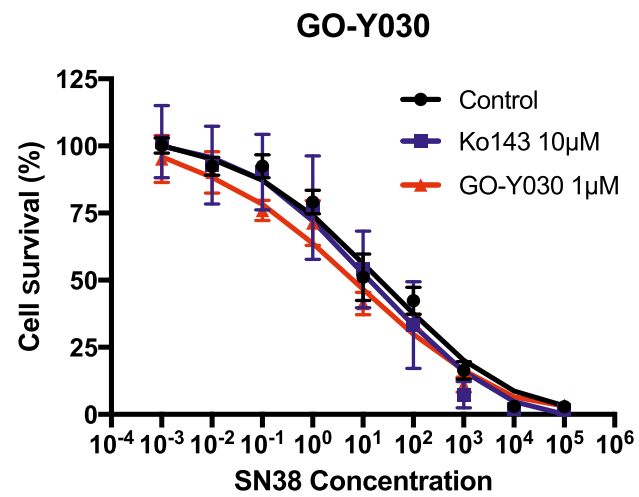

C

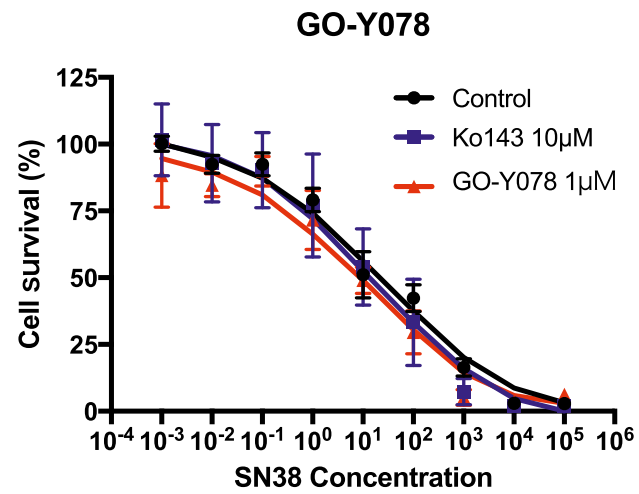

B

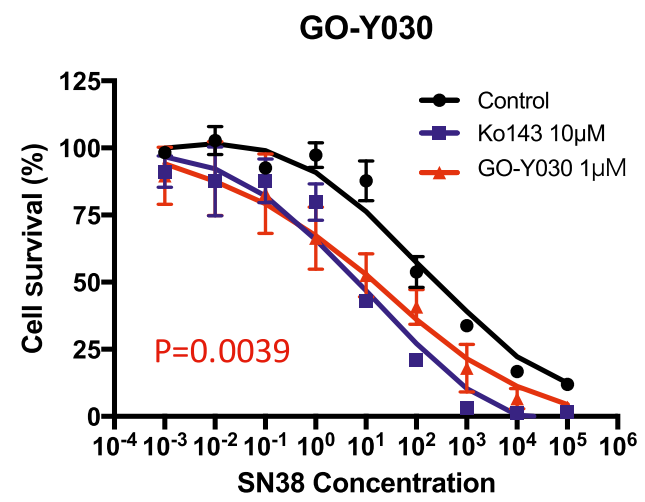

D

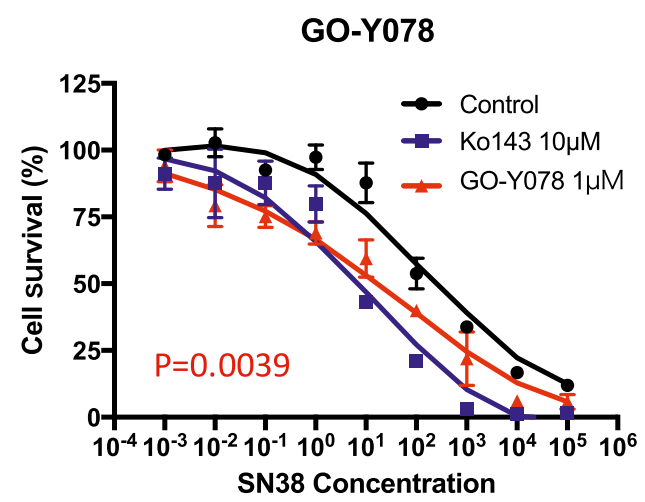

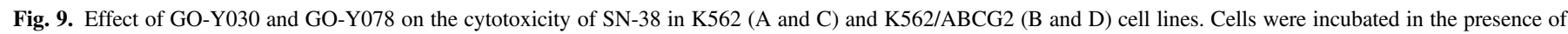

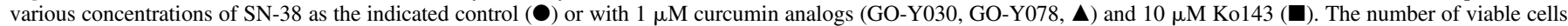

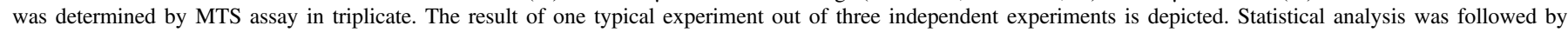
Wilcoxon matched-pairs signed rank test.

these analogs had no effect on ATP hydrolysis (data not shown). The reason why GO-Y168 showed inhibition of ATP hydrolysis at its lower concentration is unknown. It is assumed that the transporter contains two distinct binding sites for substrates. Therefore, GO-Y168 might bind to different substrate-binding sites of ABCG2 depending on its concentration. It is plausible that GO-Y168 at lower concentrations may interact with one substrate-binding site, which inhibits ATP hydrolysis; conversely, GO-Y168 at higher concentrations may bind to the other substrate-binding site, which stimulates ATP hydrolysis (McDevitt et al., 2008; Gallus et al., 2014).

We also examined the drug resistance-reversing effects of GO-Y030, GO-Y078, GO-Y168, and GO-Y172 on the cytotoxicity of SN-38 in K562 and K562/BCRP cells. Of the aforementioned analogs, GO-Y030 and GO-Y078 successfully improved the cytotoxic effect of SN-38 on the K562/BCRP cell lines. However, neither showed any cytotoxic effect on the K562 cells (Fig. 9). Therefore, GO-Y030 and GO-Y078 are considered to be effective reversal agents of ABCG2. However, it is not clear why GO-Y168 and GO-Y172 have no improvement of SN-38 sensitivity to K562/BCRP cells, whereas GO-Y168 and GO-Y172 do inhibit IAAP photolabeling and block the efflux of mitoxantrone. As the maximum ATPase activities of GO-Y168 and GO-Y172 were lower than GO-Y030 and GO-Y078 (Fig. 7), and EC 50 values of GO-Y168 and GO-Y172 were higher than GO-Y030 and GO-Y078 (Table 3), the effect of GO-Y168 and GO-Y172 might not be statistically significant.

It has been reported that GO-Y030 and GO-Y078 have shown cytotoxic effects on colon cancer cells. Of these two, GO-Y078 inhibits nuclear factor $\kappa \mathrm{B}$ transactivation, as well as the expression of the antiapoptotic proteins TP53 and DR5, more effectively than curcumin. In addition, GO-Y078 in vivo showed a 1.4-fold improvement in survival that was not matched by curcumin (Kudo et al., 2011). Therefore, the use of these analogs may possibly produce a synergistic anticancer effect by inducing the apoptosis of cancer cells and by reversing multidrug resistance with chemotherapeutic drugs. Furthermore, the solubility of GO-Y078 was two times higher than that of curcumin (Kudo et al., 2011). Thus, GO-Y078 may have a higher level of bioavailability than curcumin and could be used in clinical practice.

TABLE 4

Reversal of toxicity of SN-38 in K562/BCRP cells by curcumin analogs

Cytotoxicity assays were carried out as described in Materials and Methods, and $I_{50}$ values were determined. The values are the mean \pm S.E. of three independent experiments done in triplicates.

\begin{tabular}{lcc}
\hline \multicolumn{1}{c}{ SN-38 Treatment } & \multicolumn{1}{c}{$\mathrm{IC}_{50}$} & Fold Resistance $^{a}$ \\
\hline & $\mu M$ & \\
K562 & $9.92 \pm 0.18$ & 1.00 \\
K562/BCRP & $99.89 \pm 0.11$ & 10.07 \\
K562/BCRP + Ko143 10 $\mu \mathrm{M}$ & $9.89 \pm 0.12$ & 1.00 \\
K562/BCRP + GO-Y030 $1 \mu \mathrm{M}$ & $10.03 \pm 4.12$ & 1.01 \\
K562/BCRP + GO-Y078 $1 \mu \mathrm{M}$ & $11.71 \pm 4.14$ & 1.18 \\
\hline
\end{tabular}

${ }^{a}$ Fold resistance was determined by dividing the $\mathrm{IC}_{50}$ value of SN-38 for K562/BCRP cells with or without reversing agents by that for K562 cells. 
We also screened the curcumin analogs to identify the inhibitor for ABCB1 using flow cytometry. Although the inhibitory effect on ABCB1 by the analogs was modest, GO-Y030 and GO-Y078 showed the partial inhibition of the ABCB1-linked transporter function (data not shown). Therefore, GO-Y030 and GO-Y078 may have a joint role as a dual inhibitor of both ABCB1 and ABCG2. Recently, dual inhibitors were developed by screening based on the ABCB1 and ABCG2 docking model (Zhang et al., 2016) and the substrate common to both ABCB1 and ABCG2 (Bohn et al., 2017). It is also feasible that GO-Y030 and GO-Y078 could be used as dual inhibitors for both ABCB1- and ABCG2-expressing cancer cells.

Revalde et al. (2015) screened for ABCG2 inhibitors with curcumin analogs, the properties of which were heterocyclic cyclohexanone monocarbonyl analogs of curcumin. Their results indicated that the most effective analogs have cyclic ketone cores in place of the $\beta$-diketone found in the structure of curcumin. However, our analogs (Kohyama et al., 2015) are distinct from those analogs in terms of the terminal aromatic substituents and the structure of the central C5 linker moiety (acyclic versus cyclic). The structure-activity relationship information obtained from a panel of 24 compounds provided several useful insights into the structure and functions of the ABCG2 protein (Fig. 2). The fact that 1) four compounds (GO-Y030, GO-Y078, GO-Y168, and GO-Y172) exhibiting significant inhibitory activity against ABCG2 share bis(arylmethylidene)acetone-type cross-conjugated dienone motif with 3,5-dialkoxy substituents on the aromatic peripherals; 2) downsizing of the aromatic substituents, as found in GO-Y022 and GO-Y156, and a cyclic linker, as found in GO-Y152 and GO-Y167, led attenuation of the inhibitory activity; and 3) the half-sized derivatives (GO-Y148, GO-Y149, GO-Y150, GO-Y151, GO-Y157, GO-Y171) failed to inhibit ABCG2 activity indicated that molecules with a suitable size with moderate conformational flexibility would well find the drug-binding pocket of $\mathrm{ABCG} 2$ to interrupt its function.

C5-curcuminoids, as exemplified by GO-Y030, are shown to function as a Michael acceptor with dual active sites (Kohyama et al., 2016). Structural features of four compounds (GO-Y030, GO-Y078, GO-Y168, and GO-Y172) exhibiting significant inhibitory activity against ABCG2 may have covalent interaction(s) with nucleophilic residues (cysteine, histidine, etc.) around the binding site of $\left[{ }^{125}\right.$ I]IAAP. Elucidation of the binding mode of $\left[{ }^{125}\right.$ I]IAAP and ABCG2 will be useful to shed light on the mechanism of inhibitory activity and design of superior inhibitors.

In conclusion, this study indicates that the synthetic curcumin analogs (GO-Y030, GO-Y078, GO-Y168, and GO-Y172) can inhibit the ABCG2-mediated drug-transport function by directly interacting with the substrate-binding sites of ABCG2. Although further investigation with in vivo experiments is necessary to develop these analogs as ABCG2 inhibitors, this study suggests that curcumin analogs, especially GO-Y030 and GO-Y078, could be developed as potent modulators to address the ABCG2-mediated drug resistance of cancer cells.

\section{Acknowledgments}

The authors thank Katayama K. for providing K562 and K562/BCRP cells, Shibuya E. for general technical assistance, and the member of Department of Surgery, Tohoku University Graduate School of Medicine.

\section{Authorship Contributions}

Participated in research design: Ohnuma, Naitoh, Unno, Ambudkar. Conducted experiments: Murakami, Ohnuma, Kudoh, Ishida, Chufan.

Contributed new reagents or analytic tools: Ohnuma, Fukuda, Shibata, Iwabuchi.

Performed data analysis: Murakami, Ohnuma, Sugisawa, Kanehara, Fukuda, Iwabuchi, Ambudkar.

Wrote or contributed to the writing of the manuscript: Murakami, Ohnuma, Fukuda, Iwabuchi, Ambudkar.

\section{References}

Ambudkar SV (1998) Drug-stimulatable ATPase activity in crude membranes of human MDR1transfected mammalian cells. Methods Enzymol 292:504-514.

Ammon HP and Wahl MA (1991) Pharmacology of Curcuma longa. Planta Med 57:1-7.

Anand P, Kunnumakkara AB, Newman RA, and Aggarwal BB (2007) Bioavailability of curcumin: problems and promises. Mol Pharm 4:807-818.

Anuchapreeda S, Leechanachai P, Smith MM, Ambudkar SV, and Limtrakul PN (2002) Modulation of P-glycoprotein expression and function by curcumin in multidrug-resistant human KB cells. Biochem Pharmacol 64:573-582.

Bisht S, Feldmann G, Soni S, Ravi R, Karikar C, Maitra A, and Maitra A (2007) Polymeric nanoparticle-encapsulated curcumin ("nanocurcumin"): a novel strategy for human cancer therapy. J Nanobiotechnology 5:3

Bohn K, Lange A, Chmielewski J, and Hrycyna CA (2017) Dual modulation of human P-glycoprotein and ABCG2 with prodrug dimers of the atypical antipsychotic agent paliperidone in a model of the blood-brain barrier. Mol Pharm 14:1107-1119.

Chanmahasathien W, Ohnuma S, Ambudkar SV, and Limtrakul P (2011) Biochemical mechanism of modulation of human P-glycoprotein by stemofoline. Planta Med 77:1990-1995.

Chearwae W, Anuchapreeda S, Nandigama K, Ambudkar SV, and Limtrakul P (2004) Biochemical mechanism of modulation of human P-glycoprotein (ABCB1) by curcumin I, II, and III purified from turmeric powder. Biochem Pharmacol 68:2043-2052.

Chearwae W, Shukla S, Limtrakul P, and Ambudkar SV (2006a) Modulation of the function of the multidrug resistance-linked ATP-binding cassette transporter ABCG2 by the cancer chemopreventive agent curcumin. Mol Cancer Ther 5:1995-2006.

Chearwae W, Wu CP, Chu HY, Lee TR, Ambudkar SV, and Limtrakul P (2006b) Curcuminoids purified from turmeric powder modulate the function of human multidrug resistance protein 1 (ABCC1). Cancer Chemother Pharmacol 57:376-388.

Gallus J, Juvale K, and Wiese M (2014) Characterization of 3-methoxy flavones for their interaction with ABCG2 as suggested by ATPase activity. Biochim Biophys Acta 1838: 2929-2938.

Garcea G, Berry DP, Jones DJ, Singh R, Dennison AR, Farmer PB, Sharma RA, Steward WP, and Gescher AJ (2005) Consumption of the putative chemopreventive agent curcumin by cancer patients: assessment of curcumin levels in the colorectum and their pharmacodynamic consequences. Cancer Epidemiol Biomarkers Prev 14:120-125.

Glavinas H, Kis E, Pál A, Kovács R, Jani M, Vági E, Molnár E, Bánsághi S, Kele Z, Janáky T, et al. (2007) ABCG2 (breast cancer resistance protein/mitoxantrone resistance-associated protein) ATPase assay: a useful tool to detect drug-transporter interactions. Drug Metab Dispos 35: $1533-1542$.

Goel A, Kunnumakkara AB, and Aggarwal BB (2008) Curcumin as "curecumin": from kitchen to clinic. Biochem Pharmacol 75:787-809.

Goldberg H, Ling V, Wong PY, and Skorecki K (1988) Reduced cyclosporin accumulation in multidrug-resistant cells. Biochem Biophys Res Commun 152:552-558.

Gottesman MM, Fojo T, and Bates SE (2002) Multidrug resistance in cancer: role of ATPdependent transporters. Nat Rev Cancer 2:48-58.

Hamada H and Tsuruo T (1988) Characterization of the ATPase activity of the Mr 170,000 to 180,000 membrane glycoprotein (P-glycoprotein) associated with multidrug resistance in K562/ADM cells. Cancer Res 48:4926-4932.

Kobayashi M, Funayama R, Ohnuma S, Unno M, and Nakayama K (2016) Wnt- $\beta$-catenin signaling regulates $\mathrm{ABCC} 3$ (MRP3) transporter expression in colorectal cancer. Cancer Sci 107: 1776-1784.

Kohyama A, Fukuda M, Sugiyama S, Yamakoshi H, Kanoh N, Ishioka C, Shibata H, and Iwabuchi Y (2016) Reversibility of the thia-Michael reaction of cytotoxic C5-curcuminoid and structure-activity relationship of bis-thiol-adducts thereof. Org Biomol Chem 14:10683-10687.

Kohyama A, Yamakoshi H, Hongo S, Kanoh N, Shibata H, and Iwabuchi Y (2015) Structureactivity relationships of the antitumor C5-curcuminoid GO-Y030. Molecules 20:15374-15391.

Kudo C, Yamakoshi H, Sato A, Nanjo H, Ohori H, Ishioka C, Iwabuchi Y, and Shibata H (2011) Synthesis of 86 species of 1,5-diaryl-3-oxo-1,4-pentadienes analogs of curcumin can yield a good lead in vivo. BMC Pharmacol 11:4.

Li L, Braiteh FS, and Kurzrock R (2005) Liposome-encapsulated curcumin: in vitro and in vivo effects on proliferation, apoptosis, signaling, and angiogenesis. Cancer 104:1322-1331.

Limtrakul P, Chearwae W, Shukla S, Phisalphong C, and Ambudkar SV (2007) Modulation of function of three $\mathrm{ABC}$ drug transporters, P-glycoprotein (ABCB1), mitoxantrone resistance protein $(\mathrm{ABCG} 2)$ and multidrug resistance protein $1(\mathrm{ABCC} 1)$ by tetrahydrocurcumin, a major metabolite of curcumin. Mol Cell Biochem 296:85-95.

Lu WD, Qin Y, Yang C, Li L, and Fu ZX (2013) Effect of curcumin on human colon cancer multidrug resistance in vitro and in vivo. Clinics (Sao Paulo) 68:694-701.

Marczylo TH, Verschoyle RD, Cooke DN, Morazzoni P, Steward WP, and Gescher AJ (2007) Comparison of systemic availability of curcumin with that of curcumin formulated with phosphatidylcholine. Cancer Chemother Pharmacol 60:171-177.

McDevitt CA, Crowley E, Hobbs G, Starr KJ, Kerr ID, and Callaghan R (2008) Is ATP binding responsible for initiating drug translocation by the multidrug transporter ABCG2? FEBS J 275 : 4354-4362.

Ohori H, Yamakoshi H, Tomizawa M, Shibuya M, Kakudo Y, Takahashi A, Takahashi S, Kato S, Suzuki T, Ishioka C, et al. (2006) Synthesis and biological analysis of new curcumin analogues bearing an enhanced potential for the medicinal treatment of cancer. Mol Cancer Ther 5: 2563-2571.

Pitchakarn P, Ohnuma S, Pintha K, Pompimon W, Ambudkar SV, and Limtrakul P (2012) Kuguacin $\mathrm{J}$ isolated from Momordica charantia leaves inhibits P-glycoprotein (ABCB1)mediated multidrug resistance. $J$ Nutr Biochem 23:76-84.

Revalde JL, Li Y, Hawkins BC, Rosengren RJ, and Paxton JW (2015) Heterocyclic cyclohexanone monocarbonyl analogs of curcumin can inhibit the activity of ATP-binding cassette transporters in cancer multidrug resistance. Biochem Pharmacol 93:305-317.

Robey RW, Steadman K, Polgar O, Morisaki K, Blayney M, Mistry P, and Bates SE (2004) Pheophorbide $\mathrm{a}$ is a specific probe for ABCG2 function and inhibition. Cancer Res 64:1242-1246.

Sa G and Das T (2008) Anti cancer effects of curcumin: cycle of life and death. Cell Div 3:14.

Sauna ZE, Peng XH, Nandigama K, Tekle S, and Ambudkar SV (2004) The molecular basis of the action of disulfiram as a modulator of the multidrug resistance-linked ATP binding cassette transporters MDR1 (ABCB1) and MRP1 (ABCC1). Mol Pharmacol 65 : 675-684. 
Sharma RA, Euden SA, Platton SL, Cooke DN, Shafayat A, Hewitt HR, Marczylo TH, Morgan B, Hemingway D, Plummer SM, et al. (2004) Phase I clinical trial of oral curcumin: biomarkers of systemic activity and compliance. Clin Cancer Res 10:6847-6854

Shibata H, Yamakoshi H, Sato A, Ohori H, Kakudo Y, Kudo C, Takahashi Y, Watanabe M, Takano H, Ishioka C, et al. (2009) Newly synthesized curcumin analog has improved potential to prevent colorectal carcinogenesis in vivo. Cancer Sci 100:956-960.

Shoba G, Joy D, Joseph T, Majeed M, Rajendran R, and Srinivas PS (1998) Influence of piperine on the pharmacokinetics of curcumin in animals and human volunteers. Planta Med 64:353-356 Shukla S, Wu CP, Nandigama K, and Ambudkar SV (2007) The naphthoquinones, vitamin K3 and its structural analogue plumbagin, are substrates of the multidrug resistance linked ATP binding cassette drug transporter ABCG2. Mol Cancer Ther 6:3279-3286.

Ueda K (2011) ABC proteins protect the human body and maintain optimal health. Biosci Biotechnol Biochem 75:401-409.
Yanase K, Tsukahara S, Asada S, Ishikawa E, Imai Y, and Sugimoto Y (2004) Gefitinib reverses breast cancer resistance protein-mediated drug resistance. Mol Cancer Ther 3 : $1119-1125$.

Zhang YK, Zhang GN, Wang YJ, Patel BA, Talele TT, Yang DH, and Chen ZS (2016) Bafetinib (INNO-406) reverses multidrug resistance by inhibiting the efflux function of ABCB1 and ABCG2 transporters. Sci Rep 6:25694.

Address correspondence to: Shinobu Ohnuma, Department of Surgery, Tohoku University Graduate School of Medicine, 1-1 Seiryo-machi, Aoba-ku, Sendai, Miyagi 980-8574, Japan. E-mail: sohnuma@surg1.med.tohoku.ac.jp 